\title{
La clínica, territorio del decir trans
}

\section{The clinic, territory of the transsexual discourse}

\section{Adriana Zanón}

Correspondencia:

adrizanon@hotmail.com
Filiaciones Institucionales:

Universidad Nacional de Rosario

UNR (Argentina)

Resumen: El presente artículo se plantea indagar acerca del decir transexual en la clínica. Transexualismo, transgénero o empuje-a-la-mujer, no son conceptos, son una manera de hablar de la clínica, la que llevamos adelante en Hospital Escuela.

Se trata solamente de poder estar fieles a la clínica y escuchar lo que acontece en nuestra sociedad. Freud ha dicho que la teoría no se formula más que a posteriori y a título provisorio. Entonces, la clínica como territorio del decir trans hace a la salida de las clasificaciones psiquiátricas -de donde proviene el término transexualismo- así como de las propuestas lacanianas, las que han tenido una transmisión dogmática y religiosa. Por su parte Lacan equivocó sus apuestas, arrojando a los transexuales al campo de las psicosis o las pasiones.

Es preciso leer la clínica pluralizando el término "transexualismos" ya que se trata del decir singular, donde, en ciertos sujetos no hay delirio en juego y es bien oscilante la demanda quirúrgica de readecuación u hormonización. Entonces, hace a la variabilidad del deseo mismo, en cada momento de la existencia, según los amores, las circunstancias psíquicas, etc.

Es preciso escuchar este problema psíquico verdadero sin moralización, sino haremos otra cosa y no analizar.

PALABRAS CLAVE: clínica - transexualismo - deseo - bisexualidad

Cómo citar:

Zanón, A. (2020). La clínica, territorio del decir trans en Revista Psicoanálisis en la Universidad $\mathrm{N}^{\circ} 4$. Rosario, Argentina, UNR Editora. Pág 131-143
ISSN: 2683-9938 (en línea)

Recibido:

$27-04-2020$

Aceptado:

$03-07-2020$

Licencia: Esta obra está bajo una Licencia Creative Commons Atribución-NoComercial-Compartirlgual 4.0 Internacional.

Responsabilidad editorial:

Publicado:

$05-10-2020$ 


\section{ABSTRACT:}

This article aims to examine the transsexual discourse in the clinic. Transsexualism, transgender or push-towards-the-woman, are not concepts, they're a way to speak about the clinic, the one we carry forward at Hospital Escuela.

It consists in being faithful to the clinic and listening to what occurs in our society. Freud once said that theory is only formulated a posteriori and with provisional title. Therefore, the clinic as territory of the transsexual discourse makes a way out of the psychiatric classifications -from where the term transsexualism originated-as of Lacanian proposals, which have had a dogmatic and religious transmission. On his hand, Lacan mistook his bets, by throwing the transsexual people to the field of psychoses or passions. It's precise to read the clinic pluralizing the term "transsexualisms" as it's about the singular discourse, where, in certain subjects, there's no delirium at stake and the demand for hormonization or reassignment surgery is rather oscillating. Therefore, it alludes to the variability of desire itself, to a specific moment of existence, regarding love life, psychic circumstances, etc.

When listenning to this discourse, it's precise to avoid moralizing it, otherwise we'll be doing something else, which won't be analyzing it.

\section{KEYWORDS:}

Clinic - Transsexualism - Desire - Bisexuality. 
El sexo está vinculado al espanto. Pascal Quignard (2005, p. 57)

\section{Nuestros maestros}

En el presente artículo nos proponemos indagar acerca de los transexualismos, pluralizado el término, es decir, acerca del decir trans lo que constituye un tema eminentemente clínico. Lo proponemos entonces en términos de territorio del decir trans, sin moralización. En las curas que conducimos, si escuchamos a un analizante -en nuestros divanes o en el hospitalaquel que viene vía su decir a enunciar cuestiones acerca de lo trans, ello mismo constituye un problema psíquico verdadero, a escuchar por nuestra parte sin moralización ya que si tenemos una regla moral de antemano haremos otra cosa y no analizar. Entonces esta escritura constituye un llamado a nuestra reflexión en tanto analistas, en nuestras apuestas clínicas y teóricas en favor del psicoanálisis y su porvenir.

Hemos intentado proponer ciertas líneas de trabajo en nuestro libro de reciente publicación Transexualismos en psicosis y no psicosis. ensayo clínico (Zanón, 2019). Texto en el que plasmamos un trayecto investigativo, casos consignados y ciertos debates académicos entablados por diversos autores. Expondremos aquí algunos desarrollos sin soslayar los puntos en que tanto Freud como Lacan -es decir nuestros maestros- han ubicado sobre este tema.

El término transexualismo lo recibimos de la psiquiatría clásica. Se formuló por primera vez en la década del 50, del siglo XX. Tuvo una primera publicación en los ambientes académicos, la tesis de
Jean-Marc Alby titulada Contribution à l' étude du transsexualisme, París, 1956 (Lacan, 1987, p. 550). Pero serán Freud y Lacan los que se interesen en la lectura minuciosa de lo que nos ha testimoniado nuestro "querido presidente" Schreber -Presidente de la Corte de apelaciones de Dresde y escritor alemán- quien nos dice en sus Memorias de un enfermo nervioso (Schreber, 1999) que, se las arregló vía la Versöhnung, la reconciliación con su delirio dando paso a ser la mujer de Dios y creando una raza schreberiana. Son entonces nuestros maestros quienes recogen esta testimonialidad y le dan un estatuto propiamente analítico al transexualismo. Aunque, creemos que, tanto con Freud como con Lacan, el transexualismo ha permanecido en el campo de las psicosis, así también como con los lectores y autores de después de Lacan. Desde entonces el tema ha seguido estando en el terreno psicopatológico. Hoy estamos en el tiempo de revisar propuestas -la clínica nos lo señala- y para ello nos es preciso alejarnos de Lacan en varios puntos de su transmisión oral y escrita.

Para ello damos cuenta primero de la preciada escritura schreberiana tal como él la ha formulado en 1903, en sus Memorias publicadas. Daniel Paul Schreber escribe:

Dios nunca llegaría a una acción de retirada (con lo cual en cada oportunidad mi bienestar corporal empeora considerablemente y de inmediato), sino que la atracción se produciría sin ninguna resistencia y con un permanente equilibrio, si me fuera posible hacer siempre el papel de una mujer que yace conmigo mismo en un abrazo sexual; dejar que mi vista reposara siempre en seres 
femeninos; contemplar siempre figuras femeninas, etcétera (Schreber, 1999, p. 255). ${ }^{1}$

Este exquisito escritor de la Ilustración, admirador de la ópera, la filosofía, las letras y los tratados de psiquiatría clásica (leía y citaba a Claërembault) declara su propuesta de hombre y mujer en un abrazo constante y de un coito consigo mismo en su delirio siendo no cualquier mujer, sino la mujer del padre en su fantasma y la mujer de Dios en su delirio. Entonces, en esta gramática es el deseo del padre lo que efectúa el problema, la omnipotencia de un padre. Amar a un padre demasiado potente y que no se deja matar fantasmáticamente, simbólicamente, esto hace delirar y no la homosexualidad -lectura que llevó adelante Freud. "Es por razones del patriarcado de su época que Freud no pudo decir cuestiones. Trabajó bien el mecanismo del delirio aunque no sus causas estructurales. Entonces, lo más importante y esto lo leemos en la clínica, es que un delirio se transforma y Schreber lo testimonia en el pasaje de ser la 'puta' del doctor Fleschig a luego ser la mujer de Dios, posición más loable y de curación para nuestro sujeto.” (Pommier, 2015, p. 6)

Lacan, por su parte trabaja el tema por mojones durante su transmisión oral y escrita. Lo hace en cuatro de sus seminarios orales y en sus dos de sus Escritos. Entonces, ubicamos señalamientos en $\mathrm{El}$ Seminario 3, Las Psicosis, postulando la "transformación en mujer" importante apuesta, en El Seminario 4, La relación de objeto, en la sesión del 3 de abril de 1957 hay un esbozo de una de sus presentaciones de enfermos, un caso de "transexualismo y travestismo" donde menciona

$1 \mathrm{El}$ término "siempre" en cursiva es del texto. el "carácter desgarrador" padecido por el enfermo al ver a su hermana desnuda, aduciendo Lacan que, con esto se ilustra la perversión en su génesis.

Luego -ya en la década del 70'- en su El Seminario 18, De un discurso que no fuera del semblante, en la sesión del 20 de enero de 1971, cita a Robert Stoller (1968) en su definición de transexualismo dada en su libro Sex and Gender, la que versa: "Quizá sepan que el transexualismo consiste precisamente en un deseo muy enérgico de pasar por todos los medios al otro sexo, así sea operándose, cuando se está del lado masculino." (Lacan, 2009, p. 30). Señala que Stoller no toma en cuenta la cara psicótica de los casos descriptos ya que desconoce la forclusión lacaniana. Ubicamos aquí otro problema de Lacan, lo poco que ha hablado acerca del tema lo hizo tropezando, equivocándose al proponer el sesgo psicótico.

Por último, en El Seminario 19, ...o peor, leemos la cuestión retórica en Lacan varias veces presentada a lo largo de su transmisión oral donde dice dos cosas a la vez, dice frase tras frase y seminario tras seminario dos cosas a la vez. Transcribimos un párrafo al respecto del asunto trans:

(...) para acceder al otro sexo hay que pagar realmente el precio, el de la pequeña diferencia, que pasa engañosamente a lo real a través del órgano, debido a lo cual justamente deja de ser tomado por tal y, al mismo tiempo, revela lo que significa ser órgano. Un órgano no es instrumento más que por mediación de esto, en lo que todo instrumento se funda: que es un significante. El transexual no lo quiere en calidad de significante, y no así en calidad de órgano. En eso padece un error, que es justamente 
el error común. Su pasión, la del transexual, es la locura de querer liberarse de ese error, el error común que no ve que el significante es el goce y que el falo no es más que su significado. El transexual ya no quiere ser significado falo por el discurso sexual, que, lo enuncio, es imposible. Su único yerro es querer forzar mediante la cirugía el discurso sexual que, en cuanto imposible, es el pasaje de lo real (Lacan, 2012, p. 17).

\section{TRANSEXUALISMOS EN LA UNIVERSIDAD}

Para abordar el territorio social haremos algunas reflexiones -hoy vitales a propósito del enorme impulso que vienen cobrando las luchas, desde los diferentes sectores, así los feminismos, las minorías sexuales, las personas con discapacidad, los usuarios de la Ley Nacional de Salud Mental $N^{\circ}$ 26.657, etc.- quienes están en la línea de favorecer políticas de inclusión de los sujetos de derecho que somos todos y cada uno, desde el acompañamiento y protección que otorgan las nuevas leyes promulgadas en favor de lo que bien damos en llamar hoy políticas de ampliación de derechos sobre la base de los derechos humanos.

En lo que concierne a nuestro medio académico ha habido también avances. En 2019 y por iniciativa de nuestra Facultad de Psicología de la Universidad Nacional de Rosario UNR se promulgó la ley de cupo laboral travesti-trans para el personal no-docente universitario. La resolución lleva el nombre de "Alejandra González" en homenaje a travesti fallecida en 2018 quien desempeñó tareas no-docentes en la UNR. La misma contempla incorporar un cinco por ciento o un mínimo de tres personas por año para desempeñar tareas en diversas áreas del Rectorado y unidades académicas.

Florencia Rovetto, Secretaria de Género y sexualidad de la UNR y su equipo trabajan en favor de construir políticas inclusivas para los sujetos de la población más vulnerable con el agregado del clivaje de su orientación sexual, lo que vulnera aún más a los sujetos. En tiempos precedentes estas cuestiones se eludían... esas mismas que constituían violencias discursivas y de hecho con las consecuentes segregaciones de los denominamos confinados al gueto: "locos", "prostitutas", “maricas", desocupados y personas con discapacidad, todos ellos fuera del mundo laboral y académico.

La inclusión legal de sujetos disidentes en los cargos laborales y en la matrícula estudiantil tiene el sostenimiento de las luchas del colectivo trans, especialmente desde "La casa de las locas" de Rosario, un espacio cultural, social y político LGTBIQ+. El compromiso de ellas -las que nos resulta imposible nombrar a cada una por su nombre a los fines de este escrito- damos mención a una de sus referentes, la activista Laureana Krupp -a quien llamamos "Laly" en nuestra casa de estudios- es hoy quien ocupa el lugar de Secretaria técnica en la Secretaría de Derechos Humanos y Género de la Facultad de Psicología de la UNR bajo la dirección de Guadalupe Aguirre, ambas responsables de incorporar las perspectivas de diversidad y disidencias en la currícula de la carrera de Psicología, la que sigue ocupando un lugar de bastión del psicoanálisis y su vigencia, en nuestro país. Entonces es vital la inclusión de producción escrita y lecturas de autores LGBTIQ+ en la carrera. Los estudiantes a su vez aportan impulso e interro- 
gación a la investigación en dichas temáticas. Hay mucho por trabajar y no será sin ellos, los futuros psicólogos y/o analistas en los lugares que solemos llamar "de trinchera": Centros de Salud, Hospitales, Protocolo contra la violencia de género, etc. Dichas tareas vienen teniendo el máximo rango en el marco de la agenda en nuestra Universidad.

Y, en la égida de nuestra Provincia de Santa Fe, la Secretaría de Género promueve reuniones para los acuerdos en favor de la implementación de la Ley de Cupo Laboral Trans sancionada el 31 de octubre de 2019 por la Legislatura santafesina, la que reserva también el cinco por ciento de los ingresos al Estado provincial para personal de planta permanente, transitoria o contratada a travestis, transexuales y transgénero quienes hayan realizado la rectificación registral en sus documentos nacional de identidad.

\section{EN LA CLÍNICA}

Volvemos ahora a la clínica, territorio del decir y, formulamos que, poder nombrar les trans, los transexualismos -pluralizado el término ya que se trata de la subjetividad de cada quien-, transgénero, trans-identidades o, con Lacan empuje-a-la-mujer (pousée-à-la fermme) en su escritura guionada propuesta en su "El atolondradicho" (L'Étourdit) (Lacan, 2012, p. 490), no constituyen conceptos a propósito de los cuales sea necesario hacer un debate. Son modos de leer la clínica sin moralización: si tomamos en nuestros divanes o en el hospital en nuestras escuchas, a un sujeto que en su decir enuncia su asunción transexual, se trata de su decir singular. Enunciamos entonces que, se trata de un problema psíquico verdadero, vía el decir de cada quien; mientras que, si tenemos una regla moral de antemano en nuestras escuchas haremos otra cosa y no analizar. En este punto se torna crucial recuperar la pregunta freudiana que él escuchó y nos la legó: “¿soy hombre o soy mujer?" Pregunta irresoluble no solo en la histeria, la que podemos ubicar como la más generalizada de las neurosis, es decir la "normalidad"; la pregunta nos deja a cada sujeto hablante, cada parlêtre, cada hablante ser, tal como Lacan lo consignó a partir de los 70' al sujeto, en posición de exiliado de sí y del otro.

En este problema como en cualquier otro del psicoanálisis la teoría está siempre dispuesta a ser reemplazada, como bien la humildad freudiana la proponía. Freud claramente lo enunció respecto de sus formulaciones diciendo que estaba dispuesto a renunciar a cualquiera de sus conceptos si la experiencia así se lo indicaba. La teoría no es más que provisoria, nos decía nuestro maestro y fundador de la revolucionaria ciencia psicoanalítica.

¿Qué proponía Sigmund Freud entonces respecto de los transexualismos? Nada especial. Como lo enunciamos más arriba, el término transexualismo viene del saber psiquiátrico y se formuló recién a partir de la primera mitad del siglo veinte. Freud, por su parte, no contaba con la inmensa producción de los autores del género, no obstante es quien primero escribió acerca no sólo de la diferencia entre el sexo anatómico y el género psíquico sino que, nos dio los motivos inconscientes de dicha disyunción, con su teoría de la bisexualidad psíquica. Enorme formulación freudiana, la que atraviesa su obra de comienzo a final, desde sus "Tres ensayos para una teoría sexual" hasta "Análisis terminable e interminable.” Lo femenino y lo masculino conviven en proporción va- 
riable en cada sujeto, se entremezclan. Este es uno de los grandes legados freudianos, y el que hoy reubicamos más que nunca para entender el decir trans en nuestros análisis, haya o no demanda quirúrgica en dicho decir.

Proponemos hoy seguir a Freud y dar por fin caída a una lógica coercitiva de una sociedad caduca, la sociedad patriarcal. Por otra parte en relación con Lacan nos es preciso alejarnos de proposiciones de los años 50' donde llama "práctica transexualista" (Lacan, 1987, p. 550) a una práctica no alejada de la "perversión" según los ejemplos de observaciones que da Alby. Lacan refuerza su hipótesis diciendo que el psicótico se identifica con la carencia de ser de la madre. Y prosigue: "Sin duda la adivinación del inconsciente ha advertido muy pronto al sujeto de que, a falta de poder ser el falo que falta a la madre le queda la solución de ser la mujer que falta a los hombres" (Lacan, 1987, p. 547). ¡Discurso patriarcal si los hay! Tomaremos la tarea desde nuestra responsabilidad de analistas, la de poder salir de estas posiciones en las que Lacan se ubicó y en la que hizo recaer toda la culpa del lado de las mujeres, al ubicar la causa de las psicosis en el deseo materno, mientras que sabemos desde hace tiempo que, es por un padre incestuoso y gozador, un padre schreberiano -aunque el mismo haya sido sólo nombrado una vez a lo largo del extenso testimonio escrito- lo que está en la causa de las psicosis, más aún podríamos precisar que, en las paranoias las que constituyen la mitad de las psicosis. Solo en las esquizofrenias podemos autorizarnos a suponer una madre estrago, lo leemos en la clínica, dejando así a un hijo arrojado en el lenguaje de órgano y fragmentación corporal.
Distinguimos también aquello que Lacan llamó "transformación en mujer" en su El seminario 3, Las psicosis (Lacan, 1992, p. 439), vía la Versöhnung, la reconciliación con el delirio en el testimonio deslumbrante schreberiano, lo que será nombrado a partir de los 70 -como lo esbozamos en el comienzo- empuje-a-la-mujer (pousée-à-la femme), formulación dada en su conferencia brindada ante el equipo hospitalario en conmemoración del $50^{\circ}$ Aniversario del hospital Henri-Rousselle, en su texto llamado "El atolondradicho" (L'Étourdit) citado anteriormente. Allí nos invita a pensar que se trata de " $U n$ padre como sin razón" (Lacan, 2012, p. 490), el padre indeclinable, totémico, terrible el que produciría una risa sardónica y sarcástica, esa que para nada proviene de una alegría interior, sino, de un padre que aplasta y pasiviza fantasmáticamente a su hijo, feminizándolo. Un padre no matado fantasmáticamente.

Para concluir el recorrido lacaniano retomamos la idea de ...ou pire donde directamente nos propone que el error del transexual sería el que consiste en tratar a su órgano como significante.

En este párrafo citado en las páginas 6-7, hay una retórica a la que Lacan nos tiene acostumbrados y, se trata de la de estar diciendo dos cosas a la vez. Por ejemplo, podemos suponer que el error del transexual estaría del lado de proponer su pene como un significante, percibido -a su vez- como un órgano inútil y supernumerario. Lacan aquí menciona que "la chica" trans busca la solución quirúrgica. Y, nos preguntamos ¿acaso "el chico" trans no? Él puede llegar a buscar la readecuación de sexo, pasando a ser un varón en ciertas premisas, como lograr una erección más o menos satisfactoria, hacer pis de pie, 
etc., gracias a los mejoramientos de la técnica hormonal y/o quirúrgica de hoy día. Consultamos al respecto con especialistas -acerca de los que luego retomaremos final de este texto- ellos nos dicen que, la proporción de sujetos que desean pasar al sexo y al género masculino: "se considera uno a nueve en los casos del hospital general." ${ }^{2}$

Respecto al párrafo citado de Lacan de su El Seminario 19 ...ou pire leemos que además el transexualismo es "una pasión" o las pasiones, y, nos preguntamos ¿tal su tratamiento en Ecrits en el texto "Acerca de la causalidad psíquica”?. Más bien consideramos que es otro error de Lacan.

\section{Nuestra CLÍNICA}

A continuación daremos un fragmento de nuestra clínica sostenida en Hospital Escuela. Llamaremos G.G. a nuestro analizante quien está hospitalizado desde hace cinco años, tratado por psicosis y dependencia al alcohol, y además está abandonado a su suerte por parte de su familia. $\mathrm{Ha}$ nacido en un pequeño pueblito del norte de la provincia de Santa Fe. Luego de la muerte de sus padres, ya ancianos, sólo le queda una hermana quien padece una otra problemática psíquica y cierta posición de fragilidad.

G.G. ha conseguido hacerse una vida en el marco del hospital y del pueblo, -donde habita por el momento. En nuestro país estamos configurando las externaciones posibles -en el caso por caso- a lugares sustitutivos a la lógica manicomial. G.G. ha recorrido un trayecto en el armado de un goce singular, más viable -como bien nos decía Lacan- en su análisis. Goce

2 Dr. Daniel Lizzi responsable de las cirugías de readecuación de sexo del Hospital Centenario de Rosario. Argentina. "bien ubicado" específico, el que consiste en situarse en una posición femenina. Se siente empujado a "asumir" (más adelante retomaré este término) el lugar de mujer, porta algún detalle femenino en su presentación, usa anteojos de mujer muy llamativos, con marco de color blanco, bien femeninos. Dicho recurso es indeclinable en los momentos de salida fuera del hospital, cuando va al bar, entre otras salidas que realiza en su cotidiano vivir. Tomamos un fragmento de nuestro texto sobre transexualismos:

En entrevista él dice algo que recogemos a la letra. Es muy importante en la clínica con las psicosis que, aunque se trate de tres o cuatro frases, estas puedan ser tomadas a la letra, sin el agregado de significación posterior por parte de quien escucha. Así es como "nos dejamos aporrear por la transferencia" (expresión acuñada por Mara Musolino y por mí, en nuestro Grupo de trabajo inscripto en Convergencia: "Transferencia en las psicosis" creado en 2015), respecto de la especificidad de la transferencia psicótica. Si bien puede tratarse de un discurso deshilachado, es el decir de nuestro sujeto en el tiempo clínico en el que se encuentra tomado.

G.G. nos dice: "Tomábamos vino con mi viejo, mientras él arreglaba motos de dos tiempos en su taller. Yo no tengo físico para trabajar. Llegué aquí y desterré el vino [desde el momento de la internación hospitalaria]. Tomaba [alcohol] porque no conseguía mujer y eso me daba rabia. Rebotaba en todos lados. Los hombres, algunos sí me gustaban. Un hombre joven le vi la cola y me gustó, aunque recibí la orden de Dios que me frenó... Me pinté los labios algunas veces y vi lo mal que me quedaba, la gente me cargaba, me humillaban, me decían: 'sácate eso puto, loco"”. 
Luego agrega:

"Me doy cuenta que soy varón, que tengo barba, bellos en las piernas iy sí, mamá!”

Le señalamos:

"Escucho que le decís 'mamá' a las enfermeras, a mí y a todas las mujeres aquí."

"Sí, porque no encuentro una mujer que quiera estar conmigo."

Entonces hijo de todas, siempre hijo. (Por supuesto que esto no se lo decimos a él.)

Prosigue:

"Con los labios maquillados tuve sensación de mujer, pero sé que soy un hombre, ese es mi problema. Veo a Jael, él es 'marchanta'" (hace referencia a otro joven paciente del hospital quien se pinta las uñas y el cabello). Está ubicado en ese lugar fantasmático. Ser amado por el padre implica estar en un lugar de objeto, adormecido por el vino que se tomaban juntos, mientras sólo su padre podía trabajar. Nuestro analizante, con el sedativo del vino, soportaba ese lugar de sodomización, ocupando la posición femenina ante el padre. Es lo que pone en escena, es su mismo goce fantasmático. (Zanón, 2019, pp. 52-53-54).

Hacemos este alojamiento de un testimonio puntual acerca de lo que implica ser amado por un padre en posición de saber incestuoso, lo que nos remite a la escritura -también testimonial- la de Louis Althusser, quien, en su segunda autobiografía -escrita cinco años después del pasaje al acto homicida en que estrangula a Hélène Rytmann, su mujer- dice en El porvenir es largo: " $\mathrm{Ni}$ siquiera era un chico, sino una frágil niñita". (Althusser, 1992, p. 68) aquel quien testimonió acerca de su vida infantil bajo el sol de Argelia habiendo quedado fijado en el lugar de "nada".
Dentro del fragmento de nuestra clínica -en tanto se trata del decir singular- encontramos la variabilidad en cada decir. No hay certeza ni en las psicosis ni fuera de las psicosis acerca del decir trans. Nuestro analizante no está proponiendo la cirugía de readecuación de sexo, aunque en ciertos momentos de su análisis se ha preguntado por esta opción. Se ubica a veces como mujer, otras como varón, otras, tironeado entre ambas como desgarrado. La variabilidad de discurso en los hechos clínicos impide una adecuada ubicación en tanto que, cualquier decir fluctúa, equivoca, exilia al sujeto de sí mismo y del otro, como antes lo mencionamos.

En palabras de Pascal Quignard dadas en su libro El sexo y el espanto, escritura célebre, la que ha constituido un faro desde las letras en el avance de esta investigación, ha escrito: "El hombre no tiene el poder de permanecer erecto (...) Por eso el poder es el problema masculino por excelencia, porque su fragilidad específica y la ansiedad le preocupan a todas horas" (Quignard, 2005, p. 58).

\section{DiÁlogo CON LA CIENCIA}

Atravesamos un período social importantísimo, el que incide en nuestro trabajo en tanto analistas, ya que nuestros analizantes discurren acerca de su decir propio y a su vez, ellos viven -al igual que nosotros- en esta sociedad de hoy, la que lucha por los derechos insuficientes aún, que ejerce una virulenta violencia psíquica, plena de discursos del odio frente a las minorías sexuales, y sus políticas autónomas, así como hacia los locos del hospital históricamente confinados, segregados y a las personas con discapacidad. 
Estos discursos del odio no protegen más que a una sociedad civil sumisa. Las luchas están puestas en discurso, entonces se trata en este tema que nos ocupa acerca de les trans, que consideremos su acceso desjudicializado, declarativo y gratuito de cambio de sexo y género en el documento nacional de identidad -vigente en Argentina- así como al acceso de reembolso de los tratamientos de readecuación de sexo por parte de los sujetos que así lo deseen.

Retomamos la interlocución con el responsable de estas cirugías en el hospital público, Hospital Centenario de Rosario -el que funciona y se distingue como hospital de autogestión. Recortando las palabras médicas de nuestro texto sobre transexualismos, el Dr. Daniel Lizzi dice:

La ley es de cobertura integral y seguimiento, y es esta ley la que evita cualquier tipo de cuestionamiento de la cirugía. Agrega que las y los pacientes reciben apoyo psicológico de parte del equipo de trabajo. Luego formula cierta condición: que puedan ser escuchados en análisis por el lapso de un año aproximadamente y que por protocolo puedan mantener un año de una vida de relación con el género al cual se identifican. (Zanón, 2019, p. 93).

Después de Lacan y para abonar a la propuesta que implica una urgencia y una responsabilidad de parte nuestra, analistas, la de proseguir en la revisión de las teorías y cambiarlas si es necesario, citaré a Colette Soler en su conferencia "El empuje-a-la-mujer" dada en el Hospital Borda de Buenos Aires:

El transexual intenta hacer pasar al acto, vía la rectificación quirúrgica, la rectificación de la imagen y el estado civil. Y eso tiene un precio, sacrifican el goce. Los sujetos que se hacen operar para suprimir el órgano pene, después testimonian que no tienen ni el goce del pene ni el goce femenino, pero tienen una identificación a la mujer en la realidad. (Soler, 2011, p. 10).

¡Qué lejos que estamos hoy de estas formulaciones de las que intentamos apartarnos! La medicina actual lleva adelante cirugías que preservan la sensibilidad y el placer en el encuentro sexual en nuestros pacientes. La clínica $y$, por lo tanto, el decir singular en el territorio de la clínica, nos ubica en la vía de las palabras donde, en no pocos casos el acceso a la cirugía de readecuación de sexo y género actual, da posibilidad a la expansión subjetiva, en el momento de la vida en que un sujeto llegue a la apuesta -por la variabilidad del deseo mismo en el decir trans- a la "asunción trans”, expresión aportada por Débora Tajer, analista y feminista argentina.

En nuestro país no contamos aún con estadísticas respecto a lo que concierne a riesgos de suicidio en aquellos sujetos operados. Sí, en Francia donde el Pr. Dr. Gérard Pommier, psicoanalista en París formuló en el Prólogo de la $2^{\circ}$ edición de nuestro libro que, en diálogo con el equipo asistencial del Hospital Saint Louis -donde se realizan estas prácticas quirúrgicas- las estadísticas respecto al riesgo de suicidio no es más que la media.

\section{PARA CONCLUIR}

Ubicaremos un punto inquietante formulado a modo de pregunta: ¿El goce femenino y el goce transexual son equivalentes? Esta pregunta también la hemos discutido con el citado analista en comunicación personal, quien, generosamente nos ha 
orientado en el recorrido investigativo y escriturario de este tema eminentemente clínico. Entonces, desde su posición ética nos propuso que, nos dejemos orientar por la singularidad de la clínica, el decir singular y aproximó sencillamente la siguiente respuesta: "No es superponible tal vez el goce femenino al transexual, o por lo menos, nada se sabe hasta que el analizante hable, entonces, se espera que se diga." (Pommier, 2018).

Nos despedimos de este recorrido con una propuesta creativa la que rebasa el campo del psicoanálisis, la misma nos ha sido legada por Michel Foucault: "Devenir gay", propuesta liberadora para toda la sociedad, lo que no es equivalente a volvernos homosexuales. Él ha dicho que luego de dedicarse a la Historia de la sexualidad iba a dedicarse al problema de la amistad o las amistades tal como ha sido concebida en los siglos de la Antigüedad y subsiguientes. Enuncia:

Los sujetos disponían de alguna libertad y de cierto tipo de elección (limitada, claro está) y que también les permitía vivir relaciones afectivas intensas. La amistad tenía así mismo implicaciones económicas y sociales, el individuo estaba obligado a ayudar a sus amigos, etc. Luego en los siglos XVI y XVII desaparece este género de amistades por lo menos en la sociedad masculina. (Foucault, 1984).

La opresión patriarcal vino a desaprobar la amistad en el sentido antiguo del término.

A este respecto recogemos las palabras de un filósofo contemporáneo y activista trans, Paul B. Preciado, en su nuevo libro de reciente publicación Un departamento en Urano (Preciado, 2019, p. 101), allí formula algo elocuente, escrito además desde su propia implicación subjetiva. Lo citamos: "La transexualidad es un francotirador ciego como la risa, generoso como el amor, cariñoso y tolerante como una perra. De cuando en cuando dispara sobre un profesor de provincias o sobre una madre de familia, et boom" (Preciado, Op. cit., p. 101).

Prosigue más abajo y concluye:

Yo tenía tres años cuando sentí por primera vez el peso de la bala. Sentí que la llevaba cuando escuché a mi padre tratar de sucias tortilleras a dos chicas extranjeras que caminaban de la mano por el pueblo. Sentí en ese momento que el pecho me ardía. Esa noche, sin saber por qué, imaginé que me escapaba del pueblo para ir a un lugar extranjero. Los días que vinieron después fueron los días del miedo, de la vergüenza (Preciado, Op. cit., p. 101).

Finalmente lo decimos desde el discurso poético del genial Quignard escritor que nos acompañó y alumbró en este recorrido:

El eros es una placa arcaica, prehumana, totalmente bestial, que aborda el continente que emerge del lenguaje humano adquirido y de la vida psíquica voluntaria, adoptando las dos formas, de la angustia y de la risa. $\mathrm{La}$ angustia y la risa son las cenizas densas que caen lentamente de ese volcán. No se trata nunca del fuego abrazador ni de la roca aún incandescente y viscosa que sube del fondo de la tierra. Las sociedades y el lenguaje se protegen sin cesar de la amenaza de ese desbordamiento. En los hombres, la fabulación genealógica tiene el carácter involuntario de un reflejo muscular: son los sueños de los animales homeotermos entre- 
gados al sueño cíclico; son los mitos en las sociedades, son las novelas familiares en los individuos. Inventamos padres, es decir historias, a fin de dar sentido a lo aleatorio de un apareamiento que ninguno de nosotros -ninguno de sus frutos, tras diez oscuros meses lunares- puede ver (Quignard, Op. cit., p. 9).

En este párrafo desconcertante parece poder formularse un enlace entre la ontogénesis y la filogénesis crucial para nuestro psicoanálisis.

\section{Bibliografía}

Alby, J. (1956) Contribution à l'étude du transsexualisme, Inédita.

Allouch, J. y otros (2015) AAVV. Despatologizaciones: Homosexualidad, transexualidad...otra más?, El cuerpo queer. Subvertir la hetero-normatividad, Buenos Aires, Argentina: Letra Viva.

Althusser, L. (1992) El porvenir es largo y Los hechos, Barcelona, España: Ediciones Destino, S.A.

Czermak, M. (1987) Estudios psicoanalíticos de las psicosis. Pasiones del Objeto, Buenos Aires, Argentina: Nueva Visión.

Czermak, M. (2017) Patronymies, Considerations cliniques sur les psychoses, París. Francia: Erès.

Foucault, M. (1984) Sex, Power and the
Politics of Identity, recuperado de http:///www.foucault.devenirgays. com

Freud, S. (1979) "Nuevas conferencias de introducción al psicoanálisis", en Obras Completas, Buenos Aires, Argentina: Amorrortu, volumen (22). (1979) "Puntualizaciones psicoanalíticas sobre un caso de paranoia (Dementia paranoides) descrito autobiográficamente", en Obras Completas, Buenos Aires. Argentina: Amorrortu, volumen (12). (2010) "Análisis terminable e interminable", en Obras Completas, Buenos Aires, Argentina: Amorrortu, volumen (23).

Halperin, D. (2015) AAVV, “Cómo ser gay”, El cuerpo queer. Subvertir la hetero-normatividad, Buenos Aires, Argentina: Letra Viva.

Lacan, J. (1975) “Acerca de la causalidad psíquica”, en Escritos 1, Buenos Aires, Argentina: Siglo XXI.

(1987) "De una cuestión preliminar a todo tratamiento posible de la psicosis", en Escritos 2, Buenos Aires, Argentina: Siglo XXI.

(1992) El Seminario 3, Las psicosis, Buenos Aires, Argentina: Paidós.

(2013) El Seminario 4, La relación de objeto, Buenos Aires, Argentina: Paidós. (2006) El Seminario 23, El sinthome, Buenos Aires, Argentina: Paidós. (2009) El Seminario 18, De un discurso que no fuera del semblante, Buenos Aires, Agentina: Paidós. (2012) El Seminario 19,...o peor, Buenos Aires, Argentina: Paidós. 
(2012) "El atolondradicho", en

Otros escritos, Buenos Aires, Argentina: Paidós.

(2012) "Presentación de las memorias de un neurópata”, en Otros escritos, Buenos Aires, Argentina: Paidós.

(2013) El Seminario 4, La relación de objeto, Buenos Aires, Argentina: Paidós. (2014) "Entrevista al Sr. M. H. 26-2-76", en 8 Presentaciones de enfermos en Sainte Anne, Buenos Aires, Argentina: Editado por FFCL.

Ménard, M. (1999) Las construcciones de lo universal, Buenos Aires, Argentina: Nueva Visión.

Montrelay, M. (1970) AAVV. Investigaciones sobre la feminidad, Buenos Aires, Argentina: Nueva Visión.

Pommier, G. (1999) Louis de la Nada. La melancolía de Althusser, Buenos Aires, Argentina: Amorrrortu. (2012) ¿Qué quiere decir "hacer" el amor?, Buenos Aires, Argentina: Paidós.

(2016) Conferencia virtual “¿Cuál es el porvenir del psicoanálisis en los albores de nuestra nueva civilización?". $1^{\circ}$ Congreso Internacional de Psicoanálisis UNR. 26- 27 y 28 de Octubre de 2016. (2018) Lo femenino, una revolución sin fin, Buenos Aires, Argentina: Paidós.

Preciado, P.B. (2019) Un departamento en Urano, Buenos Aires, Argentina: Editorial Anagrama.

Quignard, P. (2005) El sexo y el espanto, Barcelona. España, Editorial Minúscula SL.
Schreber, D. P. (1999) Memorias de un enfermo nervioso, Buenos Aires, Argentina: Libros Perfil.

Soler, C. (2011) Las lecciones de las psicosis. Tres conferencias en Buenos Aires, FARP Material de circulación interna. Recuperado de http:// www. forofarp.org/images/pdf/colette2.pdf

Stoller R. (1968) Sex and Gender. The Development of Masculinity and Feminity. Reino Unido: Science House.

Zanón A. (2019) Transexualismos en psicosis y no psicosis. Ensayo clínico, Buenos Aires, Argentina: Lugar Editorial.

\section{Mg. Ps. Adriana Zanón}

Psicóloga Psicoanalista. Magister en Psicoanálisis UNR. Docente de la Cátedra Clínica II Facultad Psicología UNR. Autora de TRANSEXUALISMOS en psicosis y no-psicosis. Ensayo clínico. Lugar Editorial. 2019. Co-autora de La fábrica del caso: la sra. C. Homo Sapiens Ediciones. 1997. "Argumentos 9" Revista de la Escuela de Psicoanálisis Sigmund Freud-Rosario. 2016. Miembro AP (Analista practicante) de la Escuela de Psicoanálisis Sigmund Freud-Rosario. 